\title{
La transmisión de Conocimientos tradicionales con enfoque de género para su inclusión en la Educación ambiental
}

Lucía Martínez-Molina

Universidad de Castilla-La Mancha (España)

Carmen Solis-Espallargas

Universidad de Sevilla (España) 



\title{
La transmisión de Conocimientos tradicionales con enfoque de género para su inclusión en la Educación ambiental
}

\author{
The transmission of Traditional knowledge with a gender \\ perspective for its inclusion into environmental Education
}

\author{
Lucía Martínez-Molina \\ Universidad de Castilla-La Mancha (España) \\ lucia.martinezmolina91@gmail.com
}

Carmen Solis-Espallargas

Universidad de Sevilla (España)

carmensolise@us.es

Fecha de entrega: 17 de noviembre de 2018

Fecha de aceptación: 22 de junio de 2020

\begin{abstract}
Resumen
Históricamente los pueblos han desarrollado numerosos conocimientos útiles para la vida, saberes funcionales adaptados al territorio, compartidos y valorados por una comunidad. Su aplicación y transmisión son especialmente relevantes en la mejora de nuestra calidad de vida y la gestión los recursos naturales de una manera sostenible, especialmente en condiciones cambiantes e inciertas. Presentamos un estudio con enfoque de género e intergeneracional sobre la transmisión de Conocimientos tradicionales en la región de Andalucía Oriental en el que participaron mayores de Centros de Participación Activa y jóvenes universitarios. Cuestiones sobre práctica, origen y traspaso de Conocimientos tradicionales, así como sobre su componente sostenible han sido el centro de interés de esta investigación. Los resultados obtenidos muestran que las mujeres mayores son las principales practicantes, transmisoras y receptoras de estos conocimientos, especialmente los relacionados con la salud y los cuidados. Los datos revelan la existencia de una brecha generacional en cuanto a su práctica. El fin último de este trabajo se centra en proponer claves para fomentar la práctica y transmisión de los Conocimientos tradicionales sostenibles en programas de educación ambiental con enfoque de género.
\end{abstract}

Palabras clave: Educación Ambiental; Ecofeminismo; Género; Sostenibilidad; Conocimientos Tradicionales 


\begin{abstract}
Historically, people have developed many useful knowledge for life, functional knowledge adapted to the territory, shared and valued by a community. Its application and transmission are especially relevant to improve our quality of life and managing natural resources in a sustainable way, especially in changing and uncertain conditions.

We present a study with a gender and intergenerational approach on the transmission of Traditional knowledges in the region of Eastern Andalusia, in which elderly people from Active Participation Centers and young university students participated. Questions about practice, origin and transfer of Traditional knowledge as well as its sustainable component have been the focus of this investigation. The results show that elder women are the main practitioners, transmitters and recipients of this Knowledge, especially those related to health and care. The data reveal the existence of a generation gap in terms of its practice. The last objective will be to propose keys to promote the practice and transmission of Traditional knowledge in environmental education and gender.
\end{abstract}

Keywords: Environmental Education; Ecofeminism; Gender; Sustainability; Traditional knowledge.

\title{
1. INTRODUCGIÓN
}

A lo largo de la historia, mujeres y hombres han ido desarrollando distintos roles y responsabilidades respecto a las comunidades, hogares, familias y sus vidas debido a las estructuras sociales. Este hecho ha originado diferencias de saberes sobre los recursos naturales, diferente control y acceso a los mismos y diferentes oportunidades para participar en la toma de decisiones sobre su uso. Esta desigualdad ha provocado en muchas situaciones la invisibilización tanto de las mujeres como de la Naturaleza, así como de las labores imprescindibles para la vida que estas realizan, dando lugar a una difícil situación en la que hemos superado todas las predicciones de crecimiento poblacional y de explotación de recursos.

Debido en gran parte al pensamiento surgido en la Modernidad, vigente en la actualidad, nuestros modelos sociales se han desarrollado contrariamente a las relaciones de ecodependencia e interdependencia que aseguran el mantenimiento de la vida, por lo que arrastramos una gran crisis ambiental y una gran crisis de cuidados. Dependemos de la naturaleza, así como del tiempo y energía de otras personas puesto a disposición de los cuidados, que son universales e inevitables. La lucha común por su visibilidad se refuerza mediante los Ecofeminismos, que establecen puntos comunes entre los movimientos ecologistas y feministas. Según Puleo (2011), ofrecen al feminismo algunas claves ecológicas actuales relevantes para la lucha por la igualdad de oportunidades de las mujeres; y al ecologismo, las claves feministas que necesita para profundizar en sus planteamientos ideológicos y de acción para el cambio de modelo de desarrollo. 
En este trabajo nos centramos en investigar sobre una de estas claves, basada en la recuperación y revalorización de los saberes tradicionales sostenibles (Puleo, 2014; Huanacuni, 2010), que parecen estar perdiéndose por diferentes razones (Wolff et al., 1999; Sutherland, 2003; Sternberg et al., 2001). Por ello, pretendemos conocer desde la experiencia de las personas mayores, cómo han desarrollado la práctica y transmisión de los mismos. De igual forma, nos interesa averiguar si son conscientes de la importancia de esos conocimientos para alcanzar un modelo social que nos permita vivir de una manera más equilibrada y sostenible, todo ello desde una perspectiva de género. Este estudio se complementa con la realización de la misma investigación con personas jóvenes, para determinar la existencia o no de una brecha generacional en la transmisión de estos saberes.

En el desarrollo teórico y empírico de este trabajo nos hemos posicionado desde un planteamiento educativo en el que el factor afectivo y la necesidad de incluir los "valores ambientales y femeninos como unos valores accesibles a todos" (Novo, 2007, p.33) toman especial protagonismo, por lo que el enfoque de género resulta imprescindible. Entre los Objetivos de Desarrollo del Milenio, aprobados en la Agenda 2030 (2015), se encuentra el Objetivo 4: Educación de calidad; una de sus metas es garantizar los conocimientos necesarios para fomentar un desarrollo sostenible. Esta meta está estrechamente ligada al Objetivo 5: Igualdad de género, mostrando así la necesidad de un Educación Ambiental con perspectiva de género para conseguir un desarrollo sostenible.

Por tanto, los objetivos de este estudio se centran en conocer cuáles son los Conocimientos tradicionales más practicados con indicadores de género; evidenciar si se están perdiendo con el paso de generaciones; conocer qué valor/reconocimiento social y económico ha tenido su aplicación y si ésta es beneficiosa para la sociedad. Para ello presentamos, en una primera parte, el marco teórico y el marco metodológico sobre el cual hemos fundamentado la investigación. Finalizamos con los resultados y discusión de los mismos, así como una serie de claves para trabajar este enfoque en programas de Educación ambiental.

\section{GRISIS GLOBAL ¿CÓMO HEMOS LLEGADO HASTA AQUÍ?}

Hace ya más de 30 años el conocido informe Meadows, publicado por el Club de Roma (1972), evidenciaba la inviabilidad del crecimiento permanente de la población y sus consumos. A día de hoy los hemos sobrepasado con creces. La forma en la que las personas nos relacionamos entre nosotras y con la Naturaleza en las sociedades occidentales se opone a la organización natural del medio ambiente y la propia sociedad. Los actuales modelos de desarrollo han provocado un intenso desequilibrio en los sistemas y procesos naturales, originando así la degradación del medio ambiente y una injusta redistribución de recursos, desigualdades e injusticias sociales. Como 
dice Herrero (2013) más que ante una crisis nos encontramos ante una situación de profundo deterioro global sin precedentes.

Ante una situación tan contradictoria, cabe plantearnos cómo hemos llegado hasta aquí. Una de las razones más claras es el pensamiento occidental actual, originado en la Modernidad, época en la que surgieron nuevas concepciones acerca del mundo y el progreso presentes a día de hoy. Se desarrolló un sistema tecnocientífico que no respetaba ni los límites ni los tiempos del planeta necesarios para la vida. También se originó el sistema de pensamiento patriarcal, que presenta tres rasgos esenciales: su estructura binaria, su carácter jerárquico y su pretensión de universalidad (Herrero, 2008). Basado en una serie de dualismos (cultura/naturaleza, razón/emoción, conocimiento científico/ saber tradicional, hombre/mujer, etc.), el par cultura/naturaleza se relaciona de forma clave con el par masculino/femenino (Herrero, 2013, p.69). "Por un lado, tenemos al hombre, próximo a la cultura, la mente y la razón y por otro a la mujer, percibida como esencialmente ligada a la Naturaleza y el cuerpo y sometida a sus propias emociones".

Lagarde (1996, p.52) define el patriarcado como "un orden social genérico de poder basado en un modo de dominación cuyo paradigma es el hombre". A lo largo de la historia, este sistema, muy ligado al capitalismo en los últimos siglos, ha sometido y explotado a las mujeres y a la Naturaleza aprovechándose de sus trabajos y sus saberes, a la vez que los invisibiliza. Podemos decir que el trabajo realizado para alimentar y cuidar la vida humana a lo largo del ciclo biológico, forma ciclos repetitivos análogos a los ciclos biogeoquímicos naturales, que las sociedades occidentales no tienen en cuentan y creen poder explotar (Fernández, 2015). Este papel se ha ejercido mayoritariamente por parte de las mujeres; no porque estén biológica y naturalmente mejor formadas para ello, sino por el rol que impone la división sexual del trabajo en este tipo de sociedades. Al estudiar las dimensiones exclusivamente monetizadas, la economía convencional se organiza en torno al crecimiento económico (Brunet y Santamaría, 2016), dejando de contabilizar esos procesos, bienes y trabajos necesarios desde el punto de vista de la sostenibilidad y el bienestar.

Podemos concluir, coincidiendo con Riechmann (2012), que nos encontramos en esta situación debido fundamentalmente a dos problemas clave que articulan nuestra sociedad, afectando directamente tanto a las mujeres como a la Naturaleza: una gran crisis ambiental y una gran crisis de cuidados. Estas crisis han derivado del hecho de que los seres humanos somos seres profundamente dependientes. Por una parte, como especie, dependemos de la naturaleza, pues en ella encontramos todo lo necesario para mantenernos con vida: alimento, agua, refugio, energía...; por ello se dice que somos ecodependientes. Por otra parte, necesitamos a otros seres humanos: durante nuestro ciclo vital, dependemos materialmente del tiempo que otras personas nos dedican. Desde que nacemos hasta que morimos, nuestros cuerpos son vulnerables, envejecen y enferman, y dependemos de otros seres de nuestra misma especie para sobrevivir; por ello, se dice que somos interdependientes. Las sociedades occidentales de las que hablamos parecen no ser conscientes de estos hechos, lo cual ha hecho que se establezca 
una radical ruptura entre las personas y el resto de seres vivos, y que vivamos de espaldas a nuestra propia naturaleza, sin tener en cuenta esa doble dependencia.

\section{CONOGIMIENTOS TRADICIONALES Y SOSTENIBILIDAD}

A lo largo de la historia los pueblos han desarrollado numerosos conocimientos útiles para la vida, saberes funcionales adaptados al territorio en el que se vive. Pese a la ambigüedad del término, podemos definir los Conocimientos tradicionales - también denominados conocimiento ambiental tradicional, conocimiento ecológico local o conocimiento popular (Reyes-García, 2009) - como "el conjunto de saberes, valores, creencias y prácticas concebidas a partir de la experiencia de adaptación al entorno local a lo largo del tiempo, compartidas y valoradas por una comunidad y transmitidas de generación en generación, entendiendo por entorno local tanto el entorno cultural como el biológico" (Pardo de Santayana et al., 2014, p.20). Incluyen un espectro muy amplio de la vida de las comunidades, como uso de materiales, rasgos culturales y espirituales, conocimientos sobre plantas locales y agricultura (Eyssartier et al., 2008). Su aplicación es especialmente relevante en la mejora de nuestra calidad de vida. Aparte de su influencia en la salud y la nutrición al permitir utilizar de forma más eficiente los recursos naturales del entorno, pueden mejorar la capacidad de las sociedades para gestionar los recursos naturales, especialmente en condiciones cambiantes e inciertas (Berkes y Turner, 2006). A diferencia del conocimiento científico, estos conocimientos tradicionales son dinámicos: se transforman y van cambiando adaptándose al entorno de la comunidad, incorporando nuevos elementos y desechando otros. Para que esto pueda ocurrir, la sociedad tiene que seguir teniendo la capacidad de generar y transmitir conocimientos (Gómez-Baggethun y Reyes-García, 2013), algo que parece que se está perdiendo a nivel de comunidad debido al auge tecnológico actual.

Estudios cualitativos muestran que dicho conocimiento se pierde a medida que las comunidades se incorporan al mercado capitalista, basándose en que la entrada a la economía de mercado conlleva cambios como la realización de nuevas actividades productivas, que reducen el tiempo que las personas mantienen en contacto con la naturaleza, lo cual a su vez interfiere en la transmisión del conocimiento popular (Wolff et al., 1999). Algunos autores, como Sutherland (2003), defienden que la pérdida de diversidad cultural que implican estos conocimientos contribuye a la pérdida de diversidad biológica. Otros atribuyen la pérdida de Conocimientos tradicionales a la educación formal: al no enseñarse estos conocimientos en las escuelas, el tiempo y recursos invertidos no se destinan a ellos (Sternberg et. al., 2001). A esto se añade el hecho de que los jóvenes tienden a marcharse pronto de sus pueblos y hogares, abandonando así el modo de vida de sus mayores. Diversos estudios muestran diferencias en niveles de conocimiento entre generaciones (mayores y jóvenes). Por ejemplo, en un estudio sobre las diferencias en el conocimiento entre curanderos y no curanderos en Pichataro, México, se halló que las personas mayores tenían muchos más conocimientos que 
los jóvenes (Garro, 1986). En una investigación en Kalimanta, Indonesia, Caniago y Stephen (1998) mostraron que la gente mayor -especialmente las mujeres- tenían más conocimientos sobre plantas medicinales. La mayoría de las prácticas y relaciones con la naturaleza más sostenibles, más respetuosas e imprescindibles de recuperar se encuentran en las personas mayores.

Analizar cómo se trasmiten los Conocimientos tradicionales es de gran importancia para entender los procesos de cambio y difusión en la sociedad (Pardo de Santayana et. al., 2014). Este camino de transmisión no es simple y depende de muchos factores, como edad, género y otros factores socioculturales (Cavalli Sforza et. al., 1982). Resulta esencial identificar la participación diferenciada de mujeres y hombres tanto en la gestión como en la conservación de los recursos naturales para potenciar su participación en las comunidades.

Según Maneja (2010) existen maneras de integrar los saberes locales, históricamente apartados del discurso científico por ser juzgados como atrasados, arcaicos, primitivos o inútiles, e incorporarlos en el estudio del cambio global para plantear líneas de acción diferentes. Autores como Pretty (2003) resaltan lo conveniente que resulta crear conexiones entre diferentes redes de trabajo y grupos intergeneracionales, para promover el traspaso vertical y horizontal de los saberes.

\section{CONOCIMIENTOS TRADICIONALES Y GÉNERO}

La situación de desamparo en el que viven aún muchas mujeres de diferentes sociedades y las dificultades que continúan existiendo para ver reconocidos sus saberes y aportaciones científicas, sociales y humanas son fenómenos paralelos a la realidad de una Naturaleza que también, desde una visión patriarcal y androcéntrica, ha sido mal comprendida, no tenida en cuenta o subestimada sin que se termine por aceptar su carácter esencial de soporte de la vida y su valor para preservar la calidad de vida de las generaciones presentes y futuras.

El marco legal internacional para la protección de los conocimientos tradicionales, especialmente de pueblos indígenas, ha sido un área de gran desarrollo en años recientes. Según el informe de la UNESCO sobre Igualdad de Género, Patrimonio y Creatividad (2015, p.43) "el reconocimiento del papel de las mujeres como administradoras de la tierra y de los recursos es esencial para el éxito de las políticas de biodiversidad y patrimonio. Aunque la contribución de las mujeres en la producción agrícola es muy relevante, en especial en regiones en desarrollo, está subvalorada y subestimada en los informes". A menudo, se desconocen los trabajos realizados porque se desarrollan en el marco de la economía informal o son considerados como parte de las responsabilidades del hogar. Instituciones intergubernamentales como la FAO (Organización de las Naciones Unidas para la alimentación y la Agricultura, 2018) 
establecen que la equidad de género y el empoderamiento de las mujeres constituyen probados catalizadores para alcanzar el desarrollo sostenible.

Los diferentes posicionamientos de los Ecofeminismos - diversidad según su origen ideológico feminista y del contexto sociocultural en el que se desarrollanconfluyen en puntos comunes entendiendo que los problemas ambientales no pueden solucionarse sin enfoque de género y reclamando la imprescindible contribución de las mujeres para la construcción de otro mundo posible. Podemos decir por tanto que el Ecofeminismo es "un proyecto político, ecológico y feminista a la vez, que legitima la vida y la diversidad, y que quita legitimidad a la práctica de una cultura de la muerte que sirve de base solamente a la acumulación de capital" (Herrero, 2008, p.21), y que además nace de la convicción de que nuestro sistema "se constituyó, se ha constituido y se mantiene por medio de la subordinación de las mujeres, de la colonización de los pueblos "extranjeros" y de sus tierras, y de la Naturaleza" (Shiva y Mies, 1997). Una de las razones principales por las que la ecología se convierte en una cuestión feminista es el hecho de que la contaminación tiene particular incidencia en la salud de las mujeres y en la salud reproductiva (Puleo, 2015). Se reivindica por tanto a las mujeres como sujetos activos en la sostenibilidad ambiental y en el desarrollo de una cultura de relaciones en igualdad con la Naturaleza. Del mismo modo, debe ser reconocido su papel en la gestión de la biodiversidad y los recursos biológicos y su participación en la toma de decisiones debe asegurarse a todo nivel de la administración de los recursos.

La necesidad de esta visibilización es testimoniada por el éxito de iniciativas tales como el "I Encuentro sobre el Conocimiento Femenino para el manejo del territorio en la Amazonia Colombiana" realizado en 2017, donde se reflexionó sobre los trabajos de las mujeres, el conocimiento femenino que tienen sobre su entorno, cómo se relacionan con las plantas y los lugares del territorio que recorre día a día. En la V Asamblea de Mujeres de La Vía Campesina (2017) se defendió que el cambio global pasa indudablemente por el conocimiento de las mujeres y la soberanía alimentaria. La UNESCO llevó a cabo en el 2015 el "Proyecto para la Salvaguardia del Patrimonio Cultural Inmaterial de las Comunidades Kallawayas de Bolivia" en el que se visibilizaron los roles históricos de las mujeres como protectoras de la biodiversidad. El "Protocolo de Cooperación para Formación de Mujeres Agricultoras en Turquía" (2012-2013) empleó una estrategia sobre derechos de las mujeres y perspectivas de género en la capacitación de mujeres agrícolas rurales para reforzar sus habilidades técnicas y sus saberes sobre producción agrícola y los conocimientos tradicionales. Recientemente, se celebró en Bilbao el "XI encuentro internacional del movimiento feminista de la Marcha Mundial de las Mujeres, 2018", movimiento activo desde 1996, en el que se reflexionó sobre el contexto global y su influencia en la situación de las mujeres, centrándose este año en la necesidad de la construcción de soberanía sobre nuestros cuerpos y territorios. 
Experiencias cómo estas y la amplia diversidad de alternativas al modelo de desarrollo hegemónico son las que justifican que desde el Ecofeminismo se reivindique la necesidad del reconocimiento de los saberes tradicionales sobre las formas de aprovechamiento de los recursos, pero desde una visión más global y sostenible, primando los principios de igualdad, justicia social y corresponsabilidad socioambiental. Así, muchas mujeres trabajan por el medio ambiente aportando un planteamiento diferente, con una visión en la que se requiere que el espacio experimental, por mucho tiempo excluido a lo doméstico y privado, se expanda y amplíe sus límites hasta el mundo de lo público, donde es necesario que se revalorice la forma en la que las mujeres han venido reivindicando la relación con su medio ambiente no sólo local, sino también con perspectiva global. En muchos países las mujeres continúan con limitaciones en el acceso al aprovechamiento de los recursos naturales y a los bienes generados, soportando los costes de la explotación de esos recursos. En la mayoría de los casos, siguen siendo los hombres los que toman las decisiones en la gestión de los recursos y las mujeres siguen permaneciendo apartadas de estas cuestiones.

Estamos pues, desde prácticamente los años 70, en un momento de cambios hacia una sociedad sostenible que controle su crecimiento económico, la contaminación, la explotación de los recursos y el tamaño de su población para conseguir su mantenimiento en el tiempo y no hipotecar las posibilidades de las generaciones futuras. La posición relegada de las mujeres a lo largo de los tiempos ha sido aprovechada desde la resiliencia para poder construir trabajos en red y conocimientos relativos a la crianza, la alimentación, la salud, la agricultura, la compañía, la cohesión comunitaria, la educación y la defensa del medio natural que permita la vida, orientados hacia modelos de desarrollo más sostenibles. Por eso la sostenibilidad debe mirar, preguntar y aprender de las mujeres. Según Limón y Solís-Espallargas (2014, p.37) "Necesitamos ser conscientes de los roles y relaciones de género y de sus consecuencias sobre la construcción del mundo que queremos. Necesitamos abrir nuevos caminos que apuesten por la recuperación de saberes y el desarrollo de nuevas metodologías adaptadas a todos los grupos sociales". Es por esto que las mujeres y sus conocimientos tradicionales no pueden quedarse al margen de estos retos a los que nos enfrentamos siendo sujetos activos y visibles en esta lucha tan importante para la supervivencia humana.

\section{CONOGIMIENTOS TRADICIONALES Y EDUGACIÓN AMBIEN- TAL CON ENFOQUE DE GÉNERO}

Los problemas ambientales y sociales ante los cuales nos encontramos son resultado de acciones concretas, determinadas por los modelos de producción y consumo y por los hábitos contrarios a la vida de las sociedades occidentales. Resolver estos problemas o, mejor aún, prevenirlos, implica ir cambiando cada acción, modificando los efectos de nuestra actividad individual y colectiva, para obtener nuevas fuerzas encaminadas en una dirección distinta: la sostenibilidad de la vida. 
Pese a que la educación ambiental (en adelante EA) no puede por sí sola reemplazar la responsabilidad política ni al conocimiento científico-tecnológico, crea potencialmente las condiciones culturales apropiadas para que las problemáticas ambientales se comprendan de forma diferente. Diversas declaraciones oficiales (Belgrado, 1975; Tbilisi, 1977; Moscú 1987; Tesalónica 1997; Johannesburgo 2002, etc.) han reconocido a nivel internacional el necesario papel de la EA para poder cumplir los retos que las sociedades se han ido marcando y su orientación hacia la sostenibilidad socioambiental. En año 2015 se aprobó la Agenda 2030 por la Cumbre Especial de Desarrollo Sostenible organizada por Naciones Unidas, incluyendo 17 nuevos Objetivos de Desarrollo Sostenible (ODS). Se reconoce de nuevo la necesidad de la EA para alcanzar un desarrollo sostenible, pues el Objetivo 4: Educación de calidad, tiene entre sus metas "garantizar que todos los alumnos adquieran los conocimientos teóricos y prácticos necesarios para promover el desarrollo sostenible", mediante herramientas relacionadas con los derechos humanos, la valoración de la contribución de la cultura al desarrollo sostenible, la igualdad de género... Este último punto se corresponde con el Objetivo 5: Igualdad de género, relacionándose también de manera clave con la EA.

Quizá sea necesario empezar a decir que la EA debería "educar para pensar ambientalmente y actuar socialmente". Las comunidades tienen que ser estimuladas de una manera que construya sobre lo que tienen -incluyendo sus conocimientos tradicionales sostenibles, sus habilidades y las competencias que han adquirido a través de generaciones (Santamaría, 2008). También es tarea de la EA enseñar las alternativas disponibles a diversos temas. Así, comprender e integrar conocimientos tradicionales de agroecología y soberanía alimentaria, permacultura, comercio justo entre otros es una herramienta útil para empoderar a las personas y las comunidades. Aún continúan los programas educativos en los que los esfuerzos se centran más en comprender contenidos sobre gestión de residuos, cambio climático, contaminación y/o biodiversidad, pero con dificultades para el desarrollo de una empatía que permita una auténtica conexión con el "Otro" a pesar de esa reubicación del ser humano como parte del medio ambiente (Limón y Solís-Espallargas, 2014). La visión que guía estos programas corresponde más con un enfoque androcéntrico y antroprocéntrico que sigue sin considerar los aspectos emocionales hacia lo vivo y lo no vivo y excluye los valores femeninos al no considerarlo como objetivos para su estudio y comprensión. Entendemos que la EA no termina de superar la invisibilización de las emociones frente a la razón relacionada con antiguos dualismos. Asimismo, la limitación de la EA a la perspectiva científica de las ciencias provoca la continua separación entre lo humano y lo no humano, la reducción de la naturaleza al medio ambiente y la generalización del resto de seres vivos (Puleo, 2011). Además de la inclusión de la afectividad que nos une al otro, han de estar de forma integrada a los conocimientos científicos una educación ética crítica. "Esta plantea la inclusión de una ética del cuidado reconociendo el valor que tiene para el mantenimiento de la vida, visibilizando las aportaciones de las mujeres en condiciones de desigualdad 
y reflexionando sobre el tratamiento que desde la EA y otros medios de difusión e información ambiental se viene dando sobre ellas" (Limón y Solís-Espallargas, 2014).

Para esta tarea, la propia EA debe hacer una autocrítica respecto a los modelos educativos desde los que se sitúa, redefinir el propio concepto de medio ambiente, de ser humano y su relación con el entorno y de estrategias para la sustentabilidad. Tal vez haya que buscar nuevas respuestas que tengan que ver con la organización y el trabajo de grupos vecinales y cómo se crean los vínculos de ayuda mutua, o cómo funcionan y se mantienen las redes cooperativas agroecológicas, o cómo y quiénes son los que están realizando el cuidado de la herencia ambiental y la transmisión de conocimientos tradicionales sostenibles que recibirán las generaciones futuras, o incluso desarrollar aún más la empatía y ética con especies más allá de la humana (Limón y SolísEspallargas, 2014). Al superar el sesgo androcéntrico, la EA será más eficaz en la formación de una ciudadanía ecológicamente (co-) responsable (Puleo, 2011). Es tarea de los educadores ambientales trabajar para llegar a una sociedad en la que se aborden de una manera eficiente los problemas ambientales, y esto pasa por cambiar el modelo socioeducativo de los países desarrollados, principales responsables del deterioro del planeta.

\section{OBJETIVOS}

La finalidad de esta investigación pasa por comprender la práctica de Conocimientos tradicionales sostenibles y su posible pérdida en nuestro entorno cercano, teniendo en cuenta el papel que tienen las mujeres en esta práctica y su relación con la sostenibilidad. Por lo tanto, nos centramos en conocer cuáles son los Conocimientos tradicionales más practicados en el entorno local y analizar cómo se produce el traspaso generacional de dichos conocimientos. Así mismo, identificamos qué conocimientos tradicionales son realizados por el género femenino, para poder visibilizar el papel de las mujeres en el mantenimiento de la vida y reconocer el valor de la aplicación de esos conocimientos en el ámbito de la sostenibilidad ambiental y social. El fin último de esta investigación será considerar los resultados obtenidos sobre el análisis de la realidad de la práctica y transmisión de los conocimientos tradicionales sostenibles para que propicien la inclusión del enfoque de género en programas educativos que vengan a responder a las carencias detectadas.

\section{METODOLOGÍA DE LA INVESTIGAGIÓN}

\subsection{Muestra}

Debido al carácter intergeneracional de este trabajo, se estudiaron dos grupos poblacionales diferentes: 
Población de personas mayores: a partir de los 60 años de edad, una persona pasa a considerarse mayor, según la Organización de las Naciones Unidas (ONU, 2001). Se define esta etapa de la vida como aquella en que las capacidades individuales decrecen, en general, en comparación con las etapas anteriores, debido a la edad cronológica y según los estilos, circunstancias y ambientes de vida. Las condiciones económicas y de género también influyen en este proceso.

La población destinataria de este trabajo se centra en las personas mayores que acuden a Centros de Participación Activa (CPAs) de la Junta de Andalucía de las provincias de Málaga, Almería, Jaén y Granada, y que además participan en el Programa de Educación Ambiental Mayores por el Medio Ambiente: Recapacicla (Programa impulsado por la Consejería de Medio Ambiente de la Junta de Andalucía). Estos espacios son centros de promoción del bienestar de las personas mayores tendentes al fomento de la convivencia, la integración, la participación, la solidaridad y la relación con el medio social, pudiendo servir, sin detrimento de su finalidad esencial, de apoyo para la prestación de Servicios Sociales y Asistenciales a otros sectores de la población. Se escogió esta población debido al interés que puede tener para ella los temas planteados, como los Conocimientos tradicionales y por todo lo que se puede aprender de su experiencia. La muestra seleccionada está formada por los participantes en los Encuentros Provinciales del citado programa Recapacicla. En total se obtuvo una muestra de 52 participantes en los encuentros de las provincias de Almería, Málaga, Granada y Jaén (38 mujeres y 14 hombres), con una media de edad de 69,3 años. Aunque en la actualidad todas y todos están jubilados, la mayoría de las mujeres declararon dedicarse a sus labores o ser amas de casa, mientras que en el género masculino destacan las profesiones relacionadas con la agricultura y el sector servicios, especialmente hostelería y construcción.

Población de personas jóvenes: las definiciones sociales acerca de la juventud se reconstruyen continuamente, por eso es un concepto que nunca logra una definición estable. La juventud se entiende como una transición entre la infancia y la vida adulta. Una etapa de integración plena en la sociedad, marcada por factores socio-económicos que afectan a las oportunidades vitales de los individuos a lo largo de toda la vida (Consejería de Presidencia e Igualdad. Junta de Andalucía, 2011). Partiendo del supuesto de que se está produciendo una brecha generacional en cuanto a la transmisión de conocimientos tradicionales, el otro grupo poblacional elegido ha sido el de los jóvenes universitarios andaluces, con la intención de ponerlos en contacto con los mayores para aprender de ellas y ellos, además de poder aplicar y difundir de manera práctica estos conocimientos en su día a día. La muestra seleccionada está compuesta por estudiantes del grado de Educación primaria de la Universidad de Granada. Esta muestra fue escogida debido al futuro papel educador de los estudiantes, que tienen la capacidad de inculcar este tipo de conocimientos como docentes en formación. Además resulta muy interesante conocer su punto de vista sobre cuestiones de tipo socioambiental. Se obtuvo una muestra de 34 participantes (14 mujeres y 20 hombres) 
siendo la media de edad de 23,4 años. Respecto a la profesión, además de estudiar, cinco de los participantes simultaneaban los estudios con otros trabajos. 12 de los estudiantes proceden del medio rural y 22 del medio urbano.

\begin{tabular}{|l|c|c|c|c|}
\hline & Total & $q$ & 0 & Media de edad \\
\hline Mayores & 52 & 38 & 14 & 69,3 años \\
\hline Jóvenes & 34 & 14 & 20 & 23,4 años \\
\hline
\end{tabular}

Figura 1.- Características de la población de estudio. Ep.

\subsection{Instrumento de recogida de información}

Se realizó una investigación cuantitativa (Bisquerra, 1989) de tipo descriptivo en la que nos valimos del diseño de un cuestionario para la recogida de datos. El instrumento corresponde con un cuestionario de tipo descriptivo específico para cada grupo muestral, con preguntas cerradas no excluyentes, excepto en uno de los apartados en los que propusimos una pregunta abierta.

Para el diseño del cuestionario procedimos con una revisión bibliográfica previa con el fin de clasificar y determinar los Conocimientos tradicionales a partir del Inventario de Conocimientos tradicionales relacionados con la Biodiversidad, editado en 2014 por el Ministerio de Agricultura de España. Se hizo un primer cribado de 60 Conocimientos tradicionales y mediante consulta a grupos de expertos se seleccionaron un total de 15 Conocimientos tradicionales agrupados en categorías para facilitar el análisis (Ver tabla 1).

Tabla 1: Listado de Conocimientos tradicionales del cuestionario por categorías.

\begin{tabular}{|c|c|}
\hline \multicolumn{2}{|l|}{ A - Alimentación } \\
\hline A.1.- Cocina tradicional & $\begin{array}{l}\text { Cocina "de toda la vida", recetas familiares y sus ingredientes, } \\
\text { capacidad de alimentar a la unidad familiar día a día... }\end{array}$ \\
\hline $\begin{array}{l}\text { A.2.- Crianza de animales } \\
\text { domésticos }\end{array}$ & $\begin{array}{l}\text { Crianza de gallinas para la obtención de huevos, de cerdos para la } \\
\text { matanza... Incluyendo su alimentación y cuidados. }\end{array}$ \\
\hline A.3.- Caza y pesca & $\begin{array}{l}\text { Caza y pesca para alimentación de la unidad familiar o el uso } \\
\text { recreativo. }\end{array}$ \\
\hline \multicolumn{2}{|l|}{ B.- Salud y cuidados } \\
\hline $\begin{array}{l}\text { B.1.- Cuidado de mayores, } \\
\text { menores y personas } \\
\text { dependientes }\end{array}$ & $\begin{array}{l}\text { Ayuda a personas mayores y/o dependientes, así como a menores, } \\
\text { incluyendo higiene, vestido, seguridad, confianza, compañía, apoyo... } \\
\text { así como la educación de los hijos. }\end{array}$ \\
\hline $\begin{array}{l}\text { B.2.- Trabajo doméstico } \\
\text { rutinario }\end{array}$ & $\begin{array}{l}\text { Tareas domésticas diarias o semanales: limpieza, cuidado de la ropa } \\
\text { familiar, reparación de pequeños desperfectos, compras, etc. }\end{array}$ \\
\hline
\end{tabular}




\begin{tabular}{|c|c|}
\hline $\begin{array}{l}\text { B.3.- Remedios tradicionales } \\
\text { vinculados con la salud }\end{array}$ & $\begin{array}{l}\text { Relacionados con la salud familiar: ungüentos, remedios para fiebres } \\
\text { y resfriados, usos mágicos y/o curativos de las plantas, etc. }\end{array}$ \\
\hline \multicolumn{2}{|l|}{ C.- Medio Ambiente } \\
\hline $\begin{array}{l}\text { C.1.- Prácticas agrícolas } \\
\text { tradicionales }\end{array}$ & $\begin{array}{l}\text { Conocimientos relacionados con la agricultura tradicional: manejo de } \\
\text { un huerto familiar, uso de plantas como plaguicidas, aprovechamiento } \\
\text { de restos orgánicos para abono, bancos de semillas, etc. }\end{array}$ \\
\hline $\begin{array}{l}\text { C.2.- Conocimiento del medio } \\
\text { natural }\end{array}$ & $\begin{array}{l}\text { Reconocimiento de las especies más representativas de flora y fauna } \\
\text { de su entorno. }\end{array}$ \\
\hline $\begin{array}{l}\text { C.3.- Uso de recursos } \\
\text { naturales para la construcción } \\
\text { de herramientas y/o } \\
\text { estructuras }\end{array}$ & $\begin{array}{l}\text { Fabricación de herramientas como escobones, trillas, azadas... y/o } \\
\text { estructuras como tapiales, chamizos, etc. }\end{array}$ \\
\hline \multicolumn{2}{|l|}{ D.- Hábitos de consumo } \\
\hline D.1.- Compra local & $\begin{array}{l}\text { Compra de alimentos y productos básicos en mercados, tiendas } \\
\text { de barrio, directamente a los productores, pertenencia a redes de } \\
\text { consumo ecológico... }\end{array}$ \\
\hline D.2.- Recipientes reutilizables & $\begin{array}{l}\text { Fabricación y uso de recipientes y envoltorios reutilizables: botijos, } \\
\text { cestas, bolsas, sacos de arpillera, botellas retornables... }\end{array}$ \\
\hline D.3.- Elaboración de jabón & Reutilización de aceite usado para elaborar jabón. \\
\hline $\begin{array}{l}\text { D.4.- Construcción de objetos } \\
\text { para el juego con materiales } \\
\text { del entorno o reciclados }\end{array}$ & Muñecas con telas, coches con maderas, chapas... \\
\hline \multicolumn{2}{|l|}{ E.- Vida Social } \\
\hline $\begin{array}{l}\text { E.1.- Conocimiento de la } \\
\text { tradición oral de la zona }\end{array}$ & $\begin{array}{l}\text { Adivinanzas, cuentos, canciones infantiles, leyendas, mitos, cantos y } \\
\text { poemas épicos, sortilegios, canciones, etc. }\end{array}$ \\
\hline E.2.- Participación ciudadana & $\begin{array}{l}\text { Pertenencia y participación en asociaciones, cooperativas agrícolas, } \\
\text { clubs, sindicatos, etc. Por ejemplo: La tradición de pertenencia de una } \\
\text { familia a determinado sindicato. }\end{array}$ \\
\hline $\begin{array}{l}\text { E.3.- Toma de decisiones en la } \\
\text { vida pública }\end{array}$ & $\begin{array}{l}\text { Asistencia a debates, congresos, asambleas, juntas vecinales... y la } \\
\text { participación en la toma de decisiones. }\end{array}$ \\
\hline
\end{tabular}

Tras la selección de los principales conocimientos tradicionales se diseñaron cuatro categorías para preguntar sobre cada Conocimiento tradicional:

- Práctica: en qué medida practica ese conocimiento tradicional y cómo es su práctica. Para ello se preguntó: ¿Sabias cómo hacerlo? ¿Sabes cómo hacerlo? ¿Lo hacias? ¿Lo haces?

- Origen: ¿De quién lo aprendiste? ¿A quién veías haciéndolo comúnmente?: madre, padre, abuela, abuelo.

- Traspaso: ¿A quién lo han enseñado?: hija, hijo, nieta, nieto. 
- Sostenibilidad: En qué medida creen que ese conocimiento tradicional es más o menos sostenible (verde: sostenible, naranja: sostenibilidad media y rojo: no sostenible). Entendiendo un conocimiento tradicional sostenible como aquel cuya práctica nos ayuda a vivir de una manera más respetuosa y justa con el medio ambiente y con la sociedad (disminución de impacto ambiental, actividad para resolver problemáticas ambientales, importancia del patrimonio socioambiental).

Una vez elaborado el cuestionario se procedió a su validación por medio de expertos en la materia de diferentes universidades. Para una correcta cumplimentación del cuestionario se llevaron a cabo sesiones explicativas previas. En la siguiente Tabla 2 vemos un ejemplo del cuestionario con un ítem perteneciente a la categoría A- Alimentación:

Tabla 2: Sección del cuestionario. Ep.

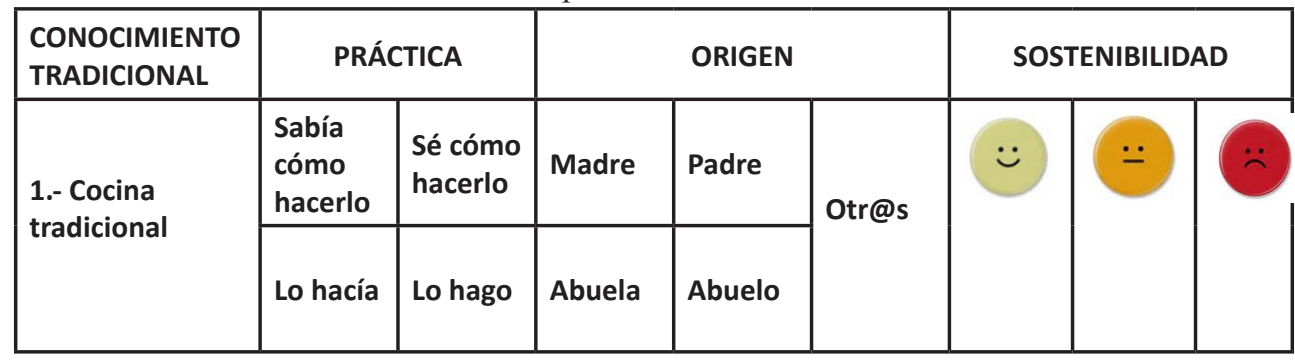

\subsection{Análisis de datos}

Para el análisis de los datos nos valimos del programa estadístico SPSS. En primer lugar, categorizamos los datos y procedimos a un análisis de respuesta múltiple utilizando tablas de contingencia. "La expresión variables de respuesta múltiple se utiliza para identificar variables en las que los sujetos pueden dar más de una respuesta, es decir, variables en las que un mismo sujeto puede tener valores distintos" (Bausela, 2005, p.69). El interés en el análisis de tablas de contingencia reside en resumir la información contenida en las mismas midiendo la asociación entre las variables consideradas, y nunca la relación entre las categorías de las variables. En este estudio, al tratarse de preguntas cerradas de tipo nominal no mutuamente excluyentes, el programa permite este tipo de análisis. Las tablas obtenidas pretenden facilitarnos el análisis respecto a la relación que se produce entre el género y la práctica/origen/ traspaso de Conocimientos tradicionales. Después de obtener los datos para cada ítem, se utilizó la función Agrupar variables para conocer el porcentaje total respecto al género, agrupando así dentro del Género femenino las respuestas dadas a las variables Madre y Abuela (para el origen) o a Hija y Nieta (para el traspaso); y a Padre y Abuelo o Hijo y Nieto para el género masculino, obteniendo así datos generales sobre el género. Tras la obtención de los 
datos definitivos se elaboraron las tablas finales a partir de las cuales se presentan los siguientes resultados.

\section{ANÁLISIS DE RESULTADOS}

a. Práctica del conocimiento tradicional

¿Sabías cómo hacerlo? ¿Sabes cómo hacerlo? ¿Lo hacias? ¿Lo haces?

Los Conocimientos tradicionales (en adelante CTs) más practicados en la muestra de Mayores son: E2.-Participación ciudadana con un 96,1\%; E1.-Tradición oral con un 94,1\%; E4.-Cooperación vecinal con un 94\%, A1.-Cocina tradicional con un $89^{\prime} 1 \%$ y E3.-Toma de decisiones en la vida pública con un $87,8 \%$. Se evidencia una predominancia de la categoría de Vida social.

En cuanto al género, los $\mathrm{CTs}$ con mayores diferencia de práctica entre mujeres y hombres son los relacionados con las categorías B.-Salud y cuidados y D.- Hábitos de consumo. Destacan los ítems más practicados por las mujeres: D3.- Elaboración de jabón con un 94\%, B3.- Remedios tradicionales 70,7\%, B2.- Trabajo doméstico rutinario $70,7 \%$ y $\mathrm{B} 1$.-Cuidado de mayores, menores y personas dependientes $60 \%$. El ítem más practicado por hombres es el A3- Caza y pesca 57,4\%. En la siguiente figura 1 se muestran (en términos de porcentajes relativos) los resultados de participantes que realizan cada CT según su género.

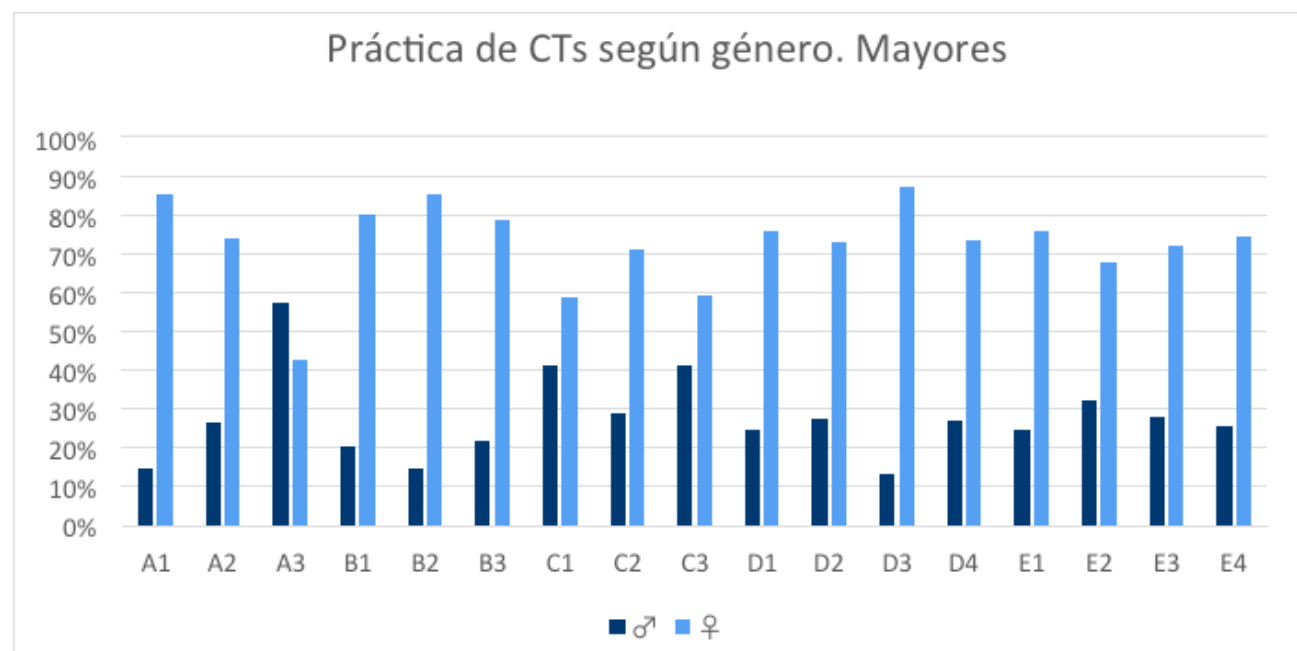

Figura 2: Práctica del CT por género en la muestra de Mayores. 
En la muestra de Jóvenes los CTs más practicados son E4.-Cooperación vecinal $81,8 \%$, B2.-Trabajo doméstico rutinario 75,8\%, C2.-Conocimiento del medio natural 63,6\% y D1.-Compra local 62,5\% y A1.-Cocina tradicional 57,6\%.

En cuanto al género, las mayores diferencias se encuentran en los CTs relacionados con Salud y cuidados: D3.-Elaboración de jabón 74,1\%, B2.-Trabajo doméstico rutinario $71 \%$, B1.-Cuidado de mayores, menores y personas dependientes $60 \%$, y B3.-Remedios tradicionales 57,2\%, En esta muestra se reduce la diferencia en ítems relacionados con Medio ambiente como A3.-Caza y pesca o A2.-Crianza de Animales domésticos. En general las mujeres jóvenes practican más los CTs que los hombres. En la figura 3 presentamos el porcentaje de participantes que practican cada CT según su género.

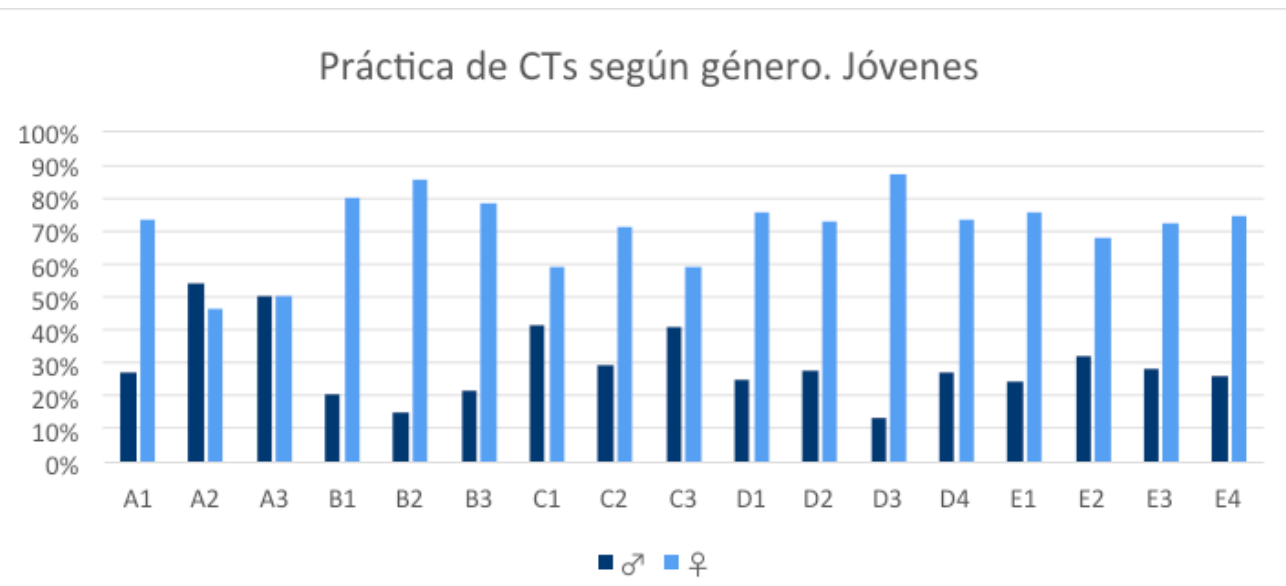

Figura 3: Práctica del CT por género en la muestra de Jóvenes.

b. Origen del aprendizaje del conocimiento tradicional

¿De quién lo aprendiste? ¿A quién veías haciéndolo comúnmente?

En la siguiente figura 4 se presentan los datos por género en los que recoge de quién aprendió cada CT, en el género femenino se incluyen madres y abuelas y en el género masculino padres y abuelos correspondiente con la muestra de Mayores.

Como se puede observar en los datos los ítems relacionados con la categoría B.- Salud y cuidados proceden en mayor medida del género femenino .-B2 Trabajo doméstico rutinario; B3.-Remedios tradicionales relacionados con la salud. Los CTs de la categoría C.-Medio ambiente proceden en su mayoría del género masculino. También es destacable el ítem A3.-Caza y pesca. 


\section{¿De quién aprendieron el CT? Mayores}

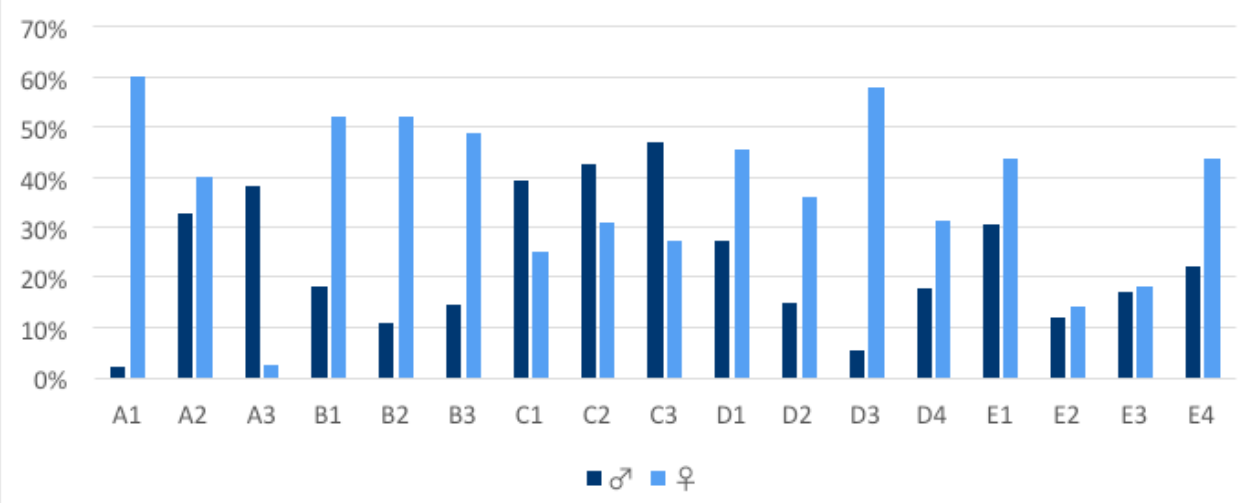

Figura 4: Origen del aprendizaje del CT por género en la muestra de Mayores.

En la muestra de Jóvenes las mayores diferencias se relacionan otra vez con Salud y cuidados (B2 «Trabajo doméstico rutinario», B3 «Remedios tradicionales»), así como Vida social (E1《Conocimiento de la tradición oral», E4 «Cooperación vecinal»).

\section{¿De quién aprendieron el CT? Jóvenes}

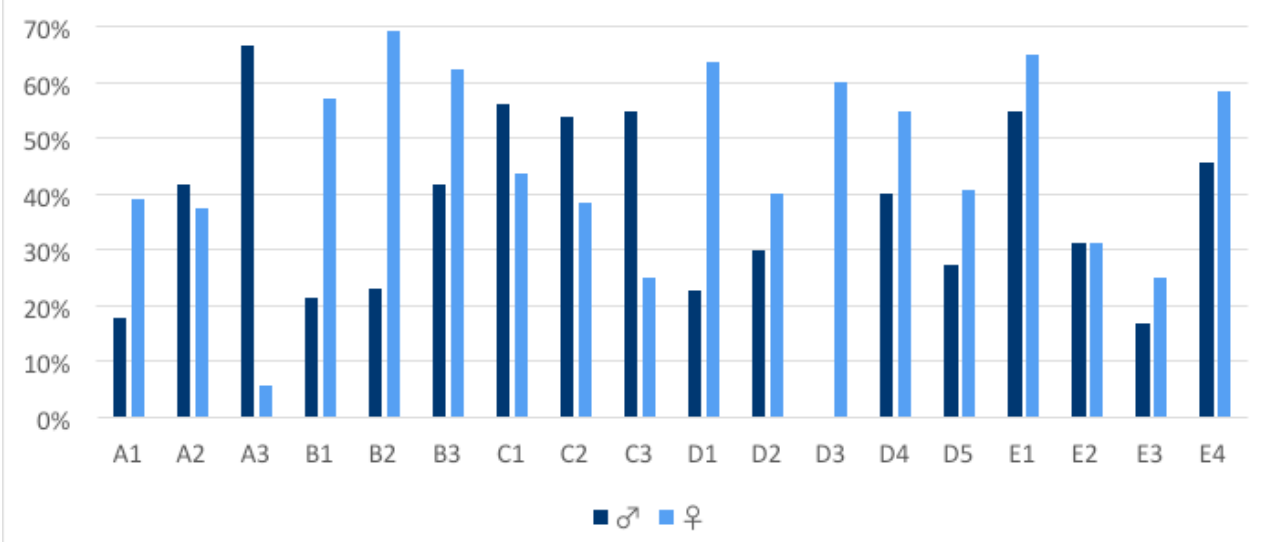

Figura 5: Origen del aprendizaje del CT por género en la muestra de Jóvenes. 
c. Transmisión de los Conocimientos tradicionales

¿A quién lo has enseñado? ¿Quién lo ha aprendido de ti?

Sobre estas cuestiones sólo se le preguntó al grupo muestral de Mayores debido al tipo de información centrada en la transmisión generacional.

Vemos como se produce un mayor traspaso al género femenino en los ítems relacionados con Saludy cuidadosy Alimentación. En cambio, en cuanto al género masculino, se produce un mayor traspaso de ítems sobre Medio ambiente, y A3.-Caza y pesca.

\section{¿A quién lo has enseñado? Mayores}

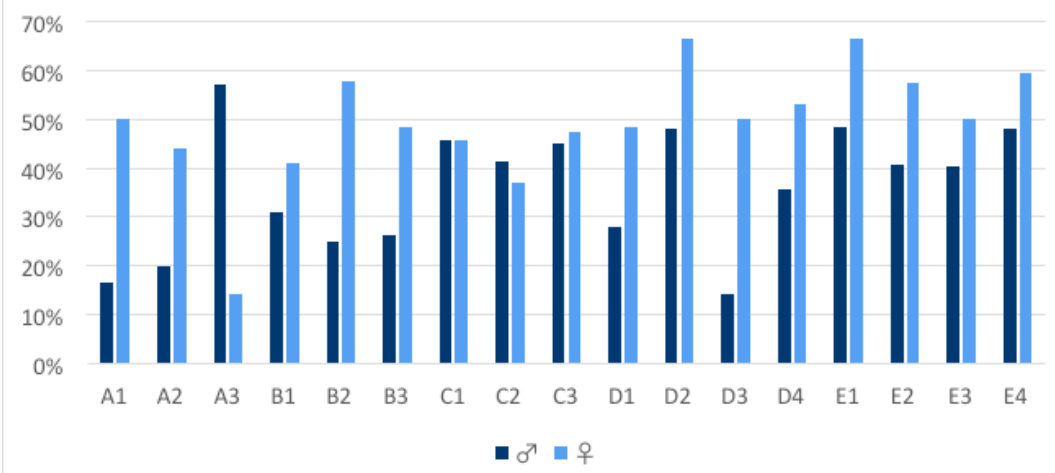

Figura 6: Traspaso del CT por género en la muestra de Mayores.

d. Deseo de aprendizaje de los conocimientos tradicionales

¿Te gustaría aprender?

Sobre estas cuestiones sólo se le preguntó al grupo muestral de Jóvenes debido al tipo de información centrada en el deseo de aprendizaje desde un enfoque generacional.
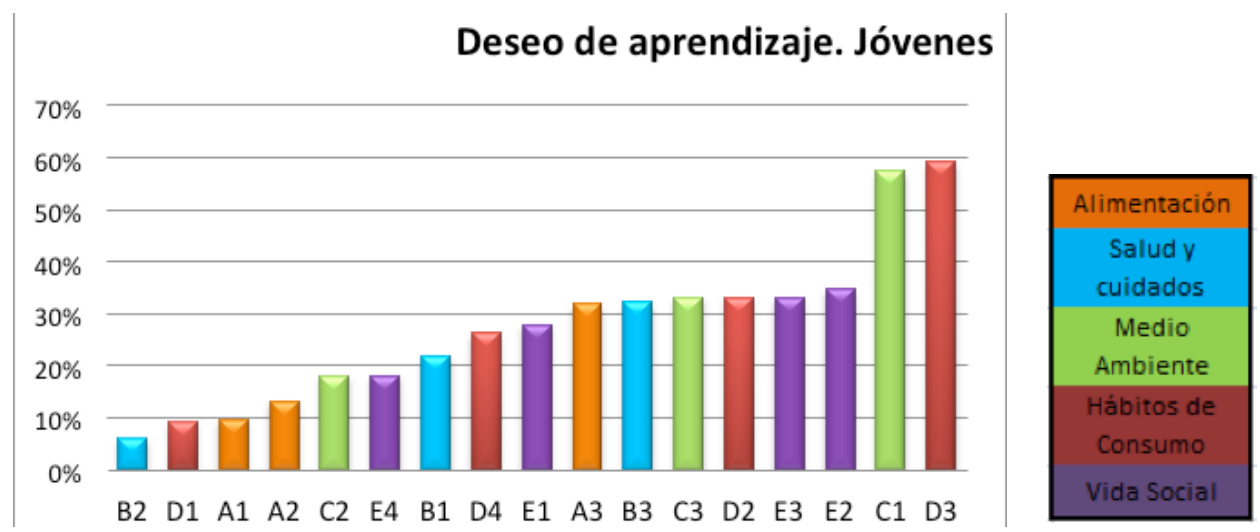

Figura 7: Deseo de aprendizaje en la muestra de Jóvenes. 
El grupo de jóvenes muestra interés por todos los ítems, pero los más relevantes están relacionados con la Vida Social: E2.-Participación ciudadana; E3.-Toma de decisiones en la vida pública, destacando C1.- Prácticas agrícolas tradicionales y D3.-Creación de jabón.

\section{e. Sostenibilidad de los Conocimientos tradicionales \\ ¿Cómo son de sostenibles los CTs?}

En las siguientes figuras se muestran de manera gráfica los CTs considerados más sostenibles (siendo aquellos que nos ayudan a vivir de una manera más respetuosa y justa con el medio ambiente y con la sociedad) por cada muestra. En la muestra Mayores destacan los ítems relacionados con Hábitos de consumo como D1.- Compra local 96,2\%, siendo el más seleccionado E2.- Participación ciudadana $98,1 \%$. En la muestra Jóvenes, podemos observar que los ítems más relevantes pertenecen a Medio ambiente: C2.- Conocimiento del medio natural 94,1\% y C3.Uso de recursos naturales $94,1 \%$.

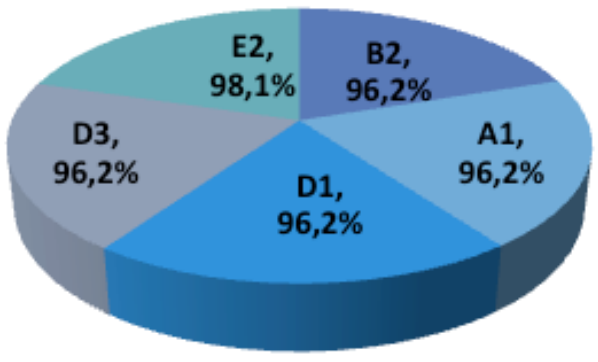

Figura 8. CTs considerados más sostenibles en la muestra Mayores.

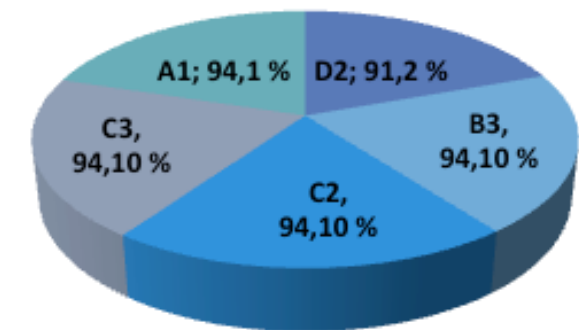

Figura 9. CTs considerados más sostenibles en la muestra Jóvenes

\section{CONGLUSIONES}

Teniendo en cuenta los resultados podemos concluir con los datos obtenidos de la muestra de Mayores y Jóvenes de este estudio que:

- Las mujeres son las que practican en mayor medida los Conocimientos tradicionales, tanto en la muestra de mayores como en la de jóvenes. Esta conclusión coincide con los resultados de diversas investigaciones y autores que confirman este hecho. Diferentes programas de organismos internacionales como la FAO o la UNESCO están apostando por programas educativos que empoderen y visibilicen a las mujeres y sus saberes tradicionales. 
- La mayor diferencia de práctica entre mujeres y hombres se encuentra en los Conocimientos tradicionales relacionados principalmente con Salud y cuidados. Esta diferencia está asociada a los roles de género heredados de las sociedades patriarcales (Herrero, 2013) en las que las mujeres continúan realizando los trabajos de atención y cuidados vitales para el sostenimiento de la vida.

- La mayoría de los encuestados aprendieron estos Conocimientos tradicionales de su madre o de su abuela, y/o los transmitieron a sus hijas o nietas. Esto nos lleva a plantear el análisis de la problemática ambiental desde una participación diferenciada de mujeres y hombres en el manejo, la degradación y la conservación de los recursos naturales así como su influencia en el traspaso de los mismos.

Por tanto, la transmisión y la práctica de los Conocimientos tradicionales continúan estando feminizados en sociedades en la que tienen una apuesta por la lucha de igualdad de oportunidades. Esta conclusión, con sus particularidades y excepciones, es extrapolable y un eje común en la diversidad de sociedades.

- En general, los encuestados de ambas muestras relacionan todos los Conocimientos tradicionales con la sostenibilidad. Las prácticas consideradas más sostenibles están relacionadas con Hábitos de consumo. La aplicación de estos conocimientos desde la sostenibilidad resulta especialmente relevante para la mejora de nuestra calidad de vida, pues permiten utilizar de forma más eficiente los recursos naturales del entorno, pudiendo proporcionar desde dietas más nutritivas hasta la prevención de enfermedades comunes (Pardo de Santayana et al., 2014). Además, mejora la capacidad de las sociedades para gestionar de una forma más eficiente los recursos naturales (Berkes y Turner, 2006).

- El ítem Caza y pesca es el único practicado por el género masculino más que por el femenino en ambas muestras. Este hecho puede deberse a que todavía perdure la idea de dominación de la Naturaleza asociada a roles de género.

-La muestra de jóvenes practica en menor medida todos los Conocimientos tradicionales. Existe por lo tanto una brecha generacional patente. Diferentes estudios (Wolff et al.,1999) confirman que estos conocimientos se pierden a medida que los grupos sociales se incorporan o crecen en el mercado capitalista. Algunos muestran diferencias en niveles de conocimiento entre generaciones (mayores y jóvenes). Otros autores (Sternberg et al; 2001) atribuyen la pérdida de conocimiento ecológico tradicional a la educación formal al no enseñarse estos conocimientos tradicionales en las escuelas. 


\section{PROPUESTAS: GLAVES PARA INCORPORAR LOS GONOGI- MIENTOS TRADICIONALES EN LA EDUCACIÓN AMBIENTAL}

La Educación Ambiental se nos presenta como una herramienta para lograr cambiar nuestras sociedades hacia modelos más sostenibles. Sería muy interesante incorporar los Conocimientos tradicionales sostenibles y su traspaso y contexto tanto en el currículo escolar como en proyectos dirigidos a otros grupos poblacionales, en especial los dirigidos a personas mayores.

La triangulación de los resultados mediante el análisis DAFO nos permite a partir de los datos realizar una planeación estratégica para la implementación de acciones y el desarrollo de proyectos de mejora (Díaz y Matamoros, 2011). Nos hemos valido del DAFO para visualizar los resultados con el fin último de este trabajo de proponer claves para una intervención educativa como podemos ver en la Tabla 3:

\begin{tabular}{|c|c|}
\hline Fortalezas & Debilidades \\
\hline $\begin{array}{l}\text { - Mayor práctica de las jóvenes del género } \\
\text { femenino en los CTs relacionados con medio } \\
\text { ambiente. } \\
\text { - Deseo de aprendizaje de los y las jóvenes. } \\
\text { - La mayoría de los encuestados de ambas } \\
\text { muestras aprendieron y transmitieron esos } \\
\text { conocimientos del y al género femenino. }\end{array}$ & $\begin{array}{l}\text { - Pérdida de práctica de CTs por parte de } \\
\text { jóvenes. Tienden a abandonar pronto } \\
\text { sus pueblos y hogares, siguiendo las } \\
\text { demandas de las sociedades occidentales } \\
\text { y abandonando así el modo de vida de sus } \\
\text { mayores. } \\
\text { - Gran diferencia de práctica de CTs } \\
\text { relacionados con Salud y cuidados en cuanto } \\
\text { al género. }\end{array}$ \\
\hline Oportunidades & Amenazas \\
\hline $\begin{array}{l}\text { - Identificar la participación diferenciada de } \\
\text { mujeres y hombres en el manejo, degradación } \\
\text { y conservación de los recursos naturales para } \\
\text { potenciar su participación en la construcción de } \\
\text { un desarrollo socioambiental sostenible. } \\
\text { - Programas educativos capaces de establecer } \\
\text { contacto intergeneracional. } \\
\text { - Revalorizar la cultura del cuidado. } \\
\text { - Revalorizar los saberes tradicionales. } \\
\text { - La aplicación de CTs mejora nuestra calidad de } \\
\text { vida. } \\
\text { - Preservar el saber que poseen las personas } \\
\text { mayores y las comunidades de todo el mundo. } \\
\text { - EA como una herramienta para encaminarnos } \\
\text { hacia un futuro sostenible. }\end{array}$ & $\begin{array}{l}\text { - Situación de profundo deterioro ecológico, } \\
\text { social y humano. } \\
\text { - En las sociedades capitalistas aquello que } \\
\text { produce beneficio económico es prioritario } \\
\text { frente a lo que beneficia a las personas. } \\
\text { - Analfabetismo ecológico. } \\
\text { - Falta de reconocimiento de las labores ejercidas } \\
\text { por el género femenino. } \\
\text { - La pérdida de diversidad cultural que implican } \\
\text { los conocimientos contribuye a la pérdida de } \\
\text { diversidad biológica a escala mundial. }\end{array}$ \\
\hline
\end{tabular}

Tabla 3.- Análisis DAFO de los resultados. Ep. 
Basándonos en el análisis DAFO realizado y en nuestros objetivos vemos fundamental el desarrollo de actividades intergeneracionales que pongan en contacto a ambas poblaciones. Sería muy interesante la promoción de encuentros entre mayores y jóvenes, en este caso aprovechando el contexto de los Centros de Participación Activa y las Universidades. En el campo de la Educación ambiental existen iniciativas encaminadas hacia estos objetivos como el programa Mayores por el medio ambiente (Consejería de Medio Ambiente y Ordenación del Territorio de la Junta de Andalucía), sin embargo, sería interesante poner el foco de atención en los Conocimientos tradicionales sostenibles y su transmisión desde una perspectiva de género e intergeneracional. Se trataría por tanto de llevar a cabo propuestas educativas para el rescate de los Conocimientos tradicionales al intercambiar experiencias, conocimientos y puntos de vista con el fin de dirigirnos a una sociedad más sostenible y un buen vivir, potenciando las relaciones de cuidados y el diálogo intergeneracional.

\section{AGRADECIMIENTOS}

Al programa Recapacicla: Mayores por el Medio Ambiente de la Consejería de Medio Ambiente y Ordenación del Territorio de la Junta de Andalucía. A todos los y las participantes del mismo. A IDEA Servicios Educativos, Culturales y Ambientales SL sin cuyo apoyo y colaboración no hubiera sido posible este estudio.

\section{BIBLIOGRAFIA}

Asher, Kiran y Varley, Gwen (2018). Gender in the jungle: a critical assessment of women and gender in current (2014-2016) forestry research. International Forestry Review, 20 (2), pp. 149-159. DOI: https://doi.org/10.1505/146554818823767537

Bausela, Esperanza (2005). SPSS: Un instrumento de análisis de datos cuantitativos. Revista de Informática Educativa y Medios Audiovisuales, 2 (4), pp. 62-69.

Berkes, Fikret y Turner, Nancy (2006). Knowledge, learning and the evolution of conservation practice for social-ecological system resilience. Human Ecology, 34 (4), pp. 479-494. DOI: https://doi.org/10.1007/s10745-006-9008-2

Bisquerra, Rafael (1989). Métodos de investigación educativa. Guía práctica. Barcelona: Ediciones CEAC.

Brunet Icart, Ignasi y Santamaría Velasco, Carlos A. (2016). La economía feminista y la división sexual del trabajo. Culturales, 4 (1), pp. 61-86.

Caniago, Izefri y Stephen, F. Siebert (1998). Medicinal plant economy, knowledge and conservation in Kalimantan. Economic Botanic, 52 (3), pp. 229-250. DOI: https:// doi.org/10.1007/BF02862141 
Cavalli Sforza, Luca L., et al. (1982). Theory and observation in cultural transmission. Science,218, (4567), pp. 19-27. DOI: https://doi.org/10.1126/science.7123211

Consejería de Presidencia e Igualdad. Junta de Andalucía (2011). Informe Social de la Juventud en Andalucía. Sevilla: Consejería de Presidencia e Igualdad de la Junta de Andalucía.

Díaz, Armando y Matamoros, Idalberto (2011). El análisis DAFO y los objetivos estratégicos. Contribuciones a la economía, 11.

Eyssartier, Cecilia; Ladio, Ana H. y Lozada, Mariana (2008). Cultural transmission of traditional knowledge in two populations of north-western Patagonia. Journal of Ethnobiology and Ethnomedicine, 4 (1), p. 25. DOI: https://doi.org/10.1186/17464269-4-25

Fernández Medrano, Hortensia (2015). Sostenibilidad y dependencias: encuentros y desencuentros entre ecologismo y feminismo. Hacia una cultura de la sostenibilidad: Análisis y propuestas desde una perspectiva de género. (E.V., Ed.). Valladolid: Departamento de Filosofía de la Universidad de Valladolid, pp. 148-153.

Fondo de las Naciones Unidas (2009). Objetivos del milenio. Informe 2008. Nueva York: Fondo de las Naciones Unidas.

Garro, Linda (1986). Intracultural variation in folk medicinal knowledge: A comparison between groups. American Anthropologist, 88 (2), pp. 351-370.

Gómez-Baggethun, Erik y Reyes-García, Victoria (2013) Reinterpreting change in traditional ecological knowledge. Human Ecology, 41, pp. 643-647. DOI: https://doi.org/10.1007/s10745-013-9577-9

Herrero, Yayo (2008). Tejer la Vida en Verde y Violeta. Vínculos entre Ecologismo y Feminismo. Cuadernos de Ecologistas en Acción. Vol. 13.

Herrero, Yayo (2013). Feminismo y ecología: Reconstruir en verde y violeta. Medio Ambiente y desarrollo. Miradas feministas desde ambos hemisferios. Granada: Universidad de Granada, pp. 67-86

Herrero, Yayo (2016). Economía feminista y economía ecológica, el diálogo necesario y urgente. Revista de Economía Crítica, 22, pp. 144-161.

Huanacuni, Fernando (2010). Buen vivir/Vivir bien. Filosofía, políticas, estrategias y experiencias regionales andinas. Perú: Coordinadora Andina de Organizaciones Indígenas-CAOI.

Lagarde, Marcela (1996). Género y feminismo: Desarrollo humano y democracia. Madrid: Horas y horas.

Limón, Dolores y Solís-Espallargas, Carmen (2014). Educación ambiental y enfoque de género: Claves para su integración. Investigación en la escuela, 83, pp. 37-50.

Maneja, Roser (2010). La percepción del Medio Ambiente en grupos infantiles y adolescentes: Comparativa entre la Huacana (Michoacán, México) y la cuenca del río Tordera (NE, Cataluña). Utopía y Praxis Latinoamericana, 14 (44), pp. 39-51. 
Meadows, Donella H. et al. (1972). The limits to growth: a report to the club of Rome. New York: Universe Books.

Memorias Encuentro sobre el Conocimiento Femenino Mujeres Indígenas de la Amazonia Colombiana Maloca de Puerto Curupira, Río Apaporis, Departamento del Vaupés-ACIYAVA. (2017). Disponible en: https://docs.wixstatic.com/ugd/ f53ce2_e262103a5f84473a8711c4d180abab77.pdf

Novo, María (2007). Mujer y medio ambiente: Los caminos de la visibilidad. Madrid: Catarata.

Organización de las Naciones Unidas (2001). Población, Medio Ambiente y Desarrollo. Informe conciso. Disponible en: http://www.sociologicamexico.azc.uam.mx/index. $\mathrm{php} /$ Sociologica/article/viewFile/450/426

Pardo de Santayana, Manuel, et al. (2014). Inventario español de los Conocimientos tradicionales relativos a la biodiversidad. Madrid: Ministerio de Agricultura, Alimentación y Medio Ambiente.

Pretty, Jules (2003). Social capital and the collective management of resources. Science, 302 (5652), pp. 1912-1914.

Puleo, Alicia (2011). Ecofeminismo para otro mundo posible. Madrid: Ediciones Cátedra.

Puleo,Alicia (2015). Iguales en un mundo sostenible. Hacia una cultura de la sostenibilidad: Análisis y propuestas desde una perspectiva de género. (E.V., Ed.). Valladolid: Departamento de Filosofía de la Universidad de Valladolid, pp. 23-38.

Reyes-García, Victoria (2009). Conocimiento ecológico tradicional para la conservación: dinámicas y conflictos. Papeles de relaciones ecosociales y cambio global, 107, pp. 39-55.

Riechmann, Jorge (2012). Interdependientes y ecodependientes: Ensayos desde la ética ecológica (y hacia ella). Barcelona: Editorial Proteus.

Santamaría, Blanca (2008): Los saberes tradicionales y la Educación ambiental: El caso de las mujeres de la cuadrilla Guadalupe Amuzgo, Oaxaca. Revista Caminos Abiertos. Disponible en: http://caminosabiertos2008.blogspot.com.es/2008/01/ los-saberes-tradicionales-y-la-educacin.html

Shiva, Vandana y Mies, Maria (1997). Ecofeminismo: teoría, crítica y perspectivas. Barcelona: Editorial Icaria.

Solís-Espallargas, Carmen (2012). Educación ambiental para el desarrollo sostenible intercultural desde un enfoque de género. (Tesis doctoral). Sevilla: Universidad de Sevilla.

Sternberg, Robert et al. (2001) The relationship between academic and practical intelligence: A case study in Kenya. Intelligence, 29 (5), pp. 401-418.

Sutherland, William J. (2003). Parallel extinction risk and global distribution of languages and species. Nature, 423 (6937), pp. 276-279.

UNESCO (2015). Igualdad de Género: Patrimonio y creatividad. UNESCO/Centro Internacional para la Promoción de los Derechos Humanos, París-Buenos Aires.

Wolff, Philip et. al. (1999). Evolution and devolution of folkbiological knowledge. Cognition, 73 (2), pp.177-204. 